\title{
CHRONIC MENINGITIS IN AN IMMUNOCOMPETENT HOST DUE TO CRYPTOCOCCUS NEOFORMANS VAR. GRUBII - FIRST CASE REPORT FROM TRIPURA, NORTH EAST INDIA
}

Tapan Majumdar', Manash Debbarma², Rakesh Das ${ }^{3}$, Pradip Bhoumik ${ }^{4}$

\section{HOW TO CITE THIS ARTICLE:}

Tapan Majumdar, Manash Debbarma, Rakesh Das, Pradip Bhoumik. "Chronic Meningitis in an Immunocompetent Host due to Cryptococcus Neoformans Var. Grubii - First Case Report from Tripura, North East India". Journal of Evolution of Medical and Dental Sciences 2015; Vol. 4, Issue 84, October 19;

Page: 14736-14740, DOI: 10.14260/jemds/2015/2096

ABSTRACT: Cryptococcosis, caused by either of two species, C. neoformans, which is characterized by haploid isolates with the A or D capsular serotype, as well as AD hybrids, or C. gattii, traditionally denoted by serotype B or C. However, more than $90 \%$ of infections worldwide are due to haploid strains of C. neoformans var. grubii, which possess the serotype A capsular epitope. Cryptococcal meningitis is mainly a disease of immunocompromised and rare in immunocompetent patients. Prognosis in immunocompetent patients is generally considered good. We report first case of cryptococcal meningitis in an immunocompetent female caused by Cryptococcus neoformans var. grubii.

KEYWORDS: Immunocompetent; Chronic meningitis; Cryptococcus neoformans var. grubi.

INTRODUCTION: Cryptococcus neoformans is an encapsulated yeast which causes life-threatening infections in approximately 2 to 3\% of patients with AIDS in the United States and in up to $40 \%$ of AIDS patients in Africa (www.who.int/hiv/; 2004 data).[1] The tropical climate of the Indian subcontinent offers a suitable environment for Cryptococcus neoformans, and the onslaught of the AIDS pandemic since the early 1990s has led to a sharp increase in the number of reported cases of cryptococcosis in the past decade.[2-4] However, more than $90 \%$ of infections worldwide are due to haploid strains of C. neoformans var. grubii, which possess the serotype A capsular epitope.[5] and majority of the Indian isolates are also serotype A and a significant minority being serotype B.[6] The inexorably neurotropic, environmental yeast, Cryptococcus neoformans var. grubii, is an opportunistic human pathogen and the leading cause of fungal meningoencephalitis . Most cases of cryptococcal disease occurs in patients who are immunocompromised though a good number cases also reported from immunocompetent patients. ${ }^{[6-7]}$

Here we report the isolation of Cryptococcus neoformans var. grubii from a young immunocompetent housewife as a first case from the State of Tripura.

CASE REPORT: A 23 years old housewife from a rural area presented with fever, frontal headache and diplopia for three days. She consulted a local physician and based on X-Ray PNS studies she was treated for maxillary sinusitis. After about seven days of treatment she again developed fever, headache, diplopia and neck stiffness for which she was referred to the tertiary care hospital. On examination patient was conscious but restless and delirious. Pulse Rate and Blood Pressure were within normal range, Temperature was $99.7^{\circ} \mathrm{F}$. There was mild pallor but no icterus and lymphadenopathy. Neck rigidity and Kernig's Sign was positive. Bilateral pupils were normal in size; reactive to light. There was evidence of left lateral rectus palsy. 
Fundoscopic examination of both eyes showed normal cup disc ratio and foveal reflex. Respiratory and Cardiovascular system was clinically normal. No past history of Tuberculosis, Diabetes Mellitus and chronic use of drugs like steroids and non-steroidal anti-inflammatory drugs. No history of any high risk behavioral activity. On laboratory Investigation, her Complete Blood Count was within normal range except raised ESR(CBC : HB-11gm\%, PCV-30, TLC-7400/cu mm of blood, DLC-N 60\%, L 38\%, E 02\%, ESR-72 1 1st $^{\text {Hr. }}$

Peripheral smear-Normocytic normochromic, Abnormal Cells-Absent). Serum Sodium$143 \mathrm{mmol} / \mathrm{dl}$, Potassium $-4.8 \mathrm{mmol} / \mathrm{dl}$, Calcium $-8.2 \mathrm{mg} / \mathrm{dl}$, Magnesium $-2.2 \mathrm{mg} / \mathrm{dl}$. Blood Urea$21 \mathrm{mg} / \mathrm{dl}$, Serum Creatinine- $0.8 \mathrm{mg} / \mathrm{dl}$, Random Blood Sugar was $92 \mathrm{mg} / \mathrm{dl}$. Liver Function Test showed- Serum Bilirubin-0.7mg/, SGOT: 26U/L, SGPT: 32U/L, Alkaline Phosphatase-128U/L. Routine urine examination was normal. Quantitative Buffy Coat Assay (QBC) for malaria parasite detection was negative. Serological Examination was negative for HIV, HCV antibody and HBsAg. Widal test was negative. Quantiferon TB Gold Test was Negative.

On radiological examination X-Ray Chest, Para Nasal sinuses were normal, Ultrasound examination of whole abdomen was within normal limit. ECG showed Sinus Tachycardia. Blood and Urine culture showed no bacterial growth. Following hospital admission she had 2-3 episode of convulsion. She was on inject able antibiotics with combination of ceftriaxone and amikacin. As there were no improvements she was put on empirical anti-tubercular drugs. After three days patient again had convulsion. Systemic steroid was added following evidence of left frontal oedema in subsequent CECT brain. At this point lumber puncture performed and CSF sent for cytological, biochemical and microbiological analysis.

Physical appearance of CSF showed clear colour without coagulam, Biochemical examination of CSF showed Protein- 16mg/dl, Sugar-31mg/dl. Cytology showed a cell count of 20/cumm, all lymphocytes. CSF ADA was found to be $06 \mathrm{U} / \mathrm{L}$. On microbiological examination: Indian Ink preparation showed plenty of encapsulated yeast cells with occasional budding (Fig.1). Gram Stain showed round yeast cells with clear halo around the cells .On ZN stain examination AFB was not found. Centrifuged deposit was cultured on Sabourad's Dextrose Agar following WHO Guideline. Mucoid colonies developed within three days of incubation at $37^{\circ} \mathrm{C}$ (Fig. 2).

Lacto Phenol Cotton Blue (LPCB) mount showed round shaped yeast cells with occasional budding. Urease test was positive (Fig. 3). Based on India Ink finding, colony character, Gram Stain finding and biochemical test results, the organism was identified as Cryptococcus species. For further confirmation and speciation the strain was sent to Centre of Advanced Research in Medical Mycology (ICMR), Department of Medical Microbiology, PGIMER, Chandigarh and was identified as Cryptococcus neoformans var grubii. Culture was negative after three weeks of antifungal therapy with amphotericinB (0.7-1.0mg/kg/day IV) and fluconazole (400mg/day PO divided doses). Patient also improved clinically with no apparent neurological deficit after six weeks of therapy. 


\section{CASE REPORT}

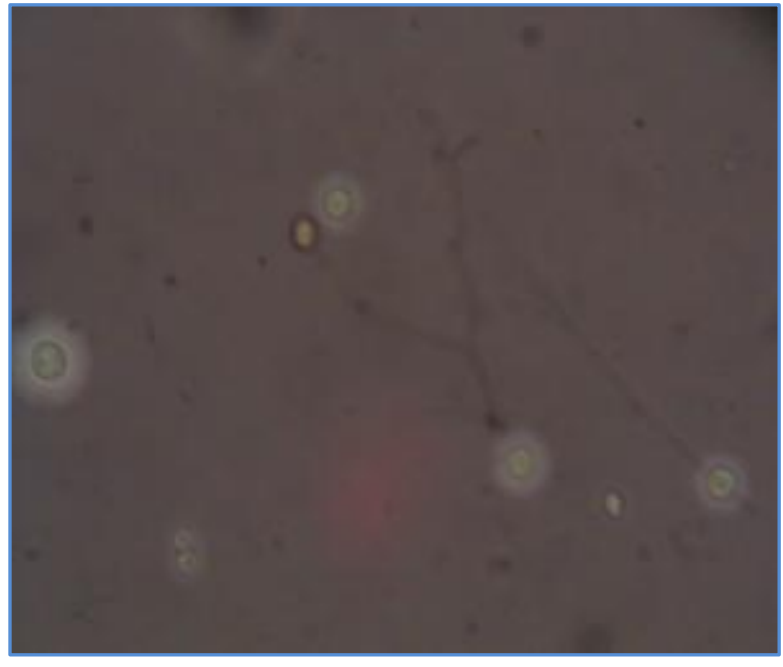

Fig. 1: Indi Ink Preparation of CSF sample showing encapsulated yeast Cells $(\mathbf{x} 400)$

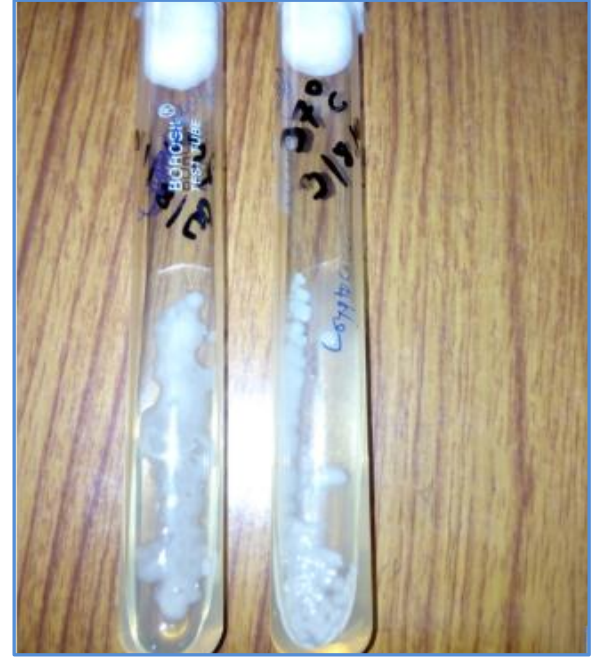

Fig. 2: Cream colored mucoid Colony in SDA with chloromphenicol incubated at $37^{\circ} \mathrm{C}$

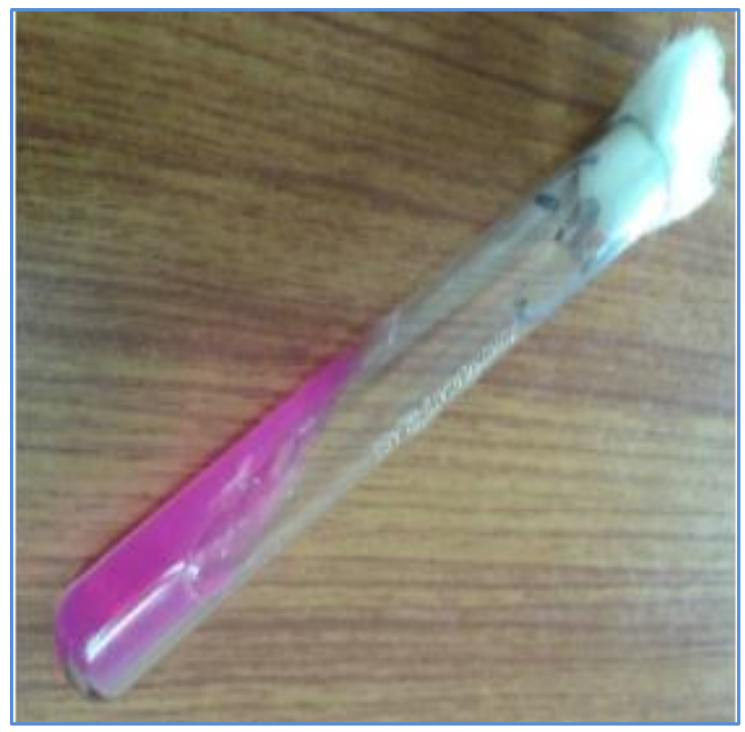

Fig. 3: Urease Test of the isolate

DISCUSSION: The patient in this case was immunocompetent and developed meningitis due to $\mathrm{C}$. neoformans var. grubii. Immunocompetent hosts are rarely reported to be infected with C.neoformans var. grubii, Whereas C.neoformans var. gattii is usually implicated, accounting for $70-80 \%$ of cryptococcal infections in such hosts. ${ }^{[8]}$ A study of the molecular epidemiology of the human pathogenic fungus C. neoformans in India has shown that most Indian isolates are $\mathrm{C}$. neoformans var. grubii (Serotype A), few are C. neoformans var. gattii (serotype B), and very few are C. neoformans var. neoformans (serotype D).[1] Prognosis in immuno-competent patient is generally considered good. But whether patient is immuno-competent or compromised, the outcome of the disease can be severe unless the disease is diagnosed early in the course of illness. 
Current practices of anti-cryptococcal therapy in India for immunocompetent patients generally include amp B alone or with flucytosine (5-fluorocytosine), and sometimes followed by fluconazole. ${ }^{[9]}$ Flucytosine was unavailable and fluconazole was started in this case. After the culture negativity fluconazole alone was continued for further six weeks.

As there was no case report from our set up this was an eye opener for the clinician. Because in this immunocompetent patient cryptococcal meningitis was never suspected and for a considerable period of time patient had received antitubercular therapy. High degree of suspicion is the need of the hour in both immunocompetent and immunocompromised patient presenting with features of chronic meningitis.

ACKNOWLEDGEMENTS: The authors wish to acknowledge Dr. Arunalok Chakraborty, Prof and Head of Centre of Advanced Research in Medical Mycology (ICMR), Department of Medical Microbiology, PGIMER, Chandigarh for his kind support in confirmation of the isolate and motivation for initiating work on mycology in this part of North east India.

\section{REFERENCES:}

1. Jain N, Wickes B L, Keller S M, Fu J, Casadevall A, Jain P et al. Molecular Epidemiology of Clinical Cryptococcus neoformans Strains from India, J Clin Microbiol, 2005; 43: 5733-42.

2. Banerjee U, Datta K, Majumdar T, Gupta K, Cryptococcosis in India: the awakening of a giant?, Med. Mycol,2001;39:51-67.

3. Banerjee U, Gupta K, Chatterjee B, Sethi S. Cryptococcosis at AIIMS, Natl. Med. J. India,1994;7:51-2.

4. Banerjee U, Khadka J B, Sethi S, Gupta K,. Sudden spurt of cryptococcosis at a tertiary care hospital at New Delhi between December 1994 to February 1995, Indian J. Med. Res, 1995; 102: 272-4.

5. Banerjee U, Datta K, Casadevall A. Serotype distribution of Cryptococcus neoformans in patients in a tertiary care centre in India. Med. Mycol. 2004; 42: 181-6.

6. Chayakulkeeree M, Perfect JR, Cryptococcosis, Infect Dis Clin North Am, 2006; 20: 507-544.

7. Idnurm A, Bahn YS, Nielsen K, Lin X, Fraser JA et al, Deciphering the model pathogenic fungus Cryptococcus neoformans. Nat Rev Microbiol, 2005; 3: 753-64.

8. Viviani MA, Tortorano AM, Ajello L, Cryptococcus. In: Clinical mycology, $1^{\text {st }}$ edition. Anaissie EJ McGinnis MR, Pfaller MA. Elsevier Science USA, 2003; 240-59.

9. Datta K, Jain N, Sethi S, Rattan A, Casadevall A, Banerjee U, Fluconazole and itraconazole susceptibility of clinical isolates of Cryptococcus neoformans at a tertiary care centre in India: a need for care. J Antimicrob Chemother, 2003; 52: 683-6. 


\section{CASE REPORT}

\section{AUTHORS:}

1. Tapan Majumdar

2. Manash Debbarma

3. Rakesh Das

4. Pradip Bhoumik

\section{PARTICULARS OF CONTRIBUTORS:}

1. Associate Professor, Department of Microbiology, Agartala Government Medical College.

2. Resident Doctor, Department of Microbiology, Agartala Government Medical College.

3. Resident Doctor, Department of Medicine, Agartala Government Medical College.

FINANCIAL OR OTHER

COMPETING INTERESTS: None
4. Associate Professor, Department of Medicine, Agartala Government Medical College.

\section{NAME ADDRESS EMAIL ID OF THE} CORRESPONDING AUTHOR:

Dr. Tapan Majumdar, Associate Professor, Department of Microbiology, Agartala Government Medical College, P.0., Kunjavan-799006,

Tripura (West).

E-mail: drtapan1@rediffmail.com

Date of Submission: 28/09/2015. Date of Peer Review: 29/09/2015. Date of Acceptance: 09/10/2015. Date of Publishing: 19/10/2015. 